\title{
Assessment of a multi-marker risk score for predicting cause-specific mortality at three years in older patients with heart failure and reduced ejection fraction
}

\author{
Christian Bjurman ${ }^{1}$, Alexandra Holmström ${ }^{1}$, Max Petzold ${ }^{2}$, \\ Ola Hammarsten ${ }^{3}$, Michael Lx Fu ${ }^{1}$ \\ ${ }^{1}$ Institute of Medicine, Sahlgrenska University Hospital, \\ Sahlgrenska Academy at University of Gothenburg, Gothenburg, Sweden \\ ${ }^{2}$ Center for Applied Biostatistics, Occupational and Environmental Medicine, \\ The Sahlgrenska Academy at University of Gothenburg, Gothenburg, Sweden \\ ${ }^{3}$ Department of Clinical Chemistry and Transfusion Medicine, Institute of Biomedicine, \\ The Sahlgrenska Academy at University of Gothenburg, Gothenburg, Sweden
}

\begin{abstract}
Background: Due to increasing co-morbidity associated with aging, heart failure (HF) has become more prevalent and heterogeneous in older individuals, and non-cardiovascular (CV) mortality has increased. Previously, we defined a multi-marker modality that included cystatin $C$ (CysC), troponin $T(T n T)$, and age. Here, we validated this multi-marker risk score by evaluating its predictions of all-cause mortality and CV mortality in an independent population of older individuals with $H F$ and reduced ejection fraction (HFrEF).

Methods: This prospective cohort study included 124 patients, median age 73 years, that had HFrEF. We determined all-cause mortality and CV mortality at a 3-year follow-up. We compared the risk score to the $N$-terminal prohormone of $B$-type natriuretic peptide (NT-proBNP) for predicting all-cause mortality and $C V$ mortality.

Results: High risk scores were associated with both all-cause mortality (HR 4.2, 95\% CI 2.2-8.1, $p<0.001$ ) and CV mortality (HR 3.6, 95\% CI 1.7-8.0, $p=0.0015)$. Receiver operating characteristics showed similar efficacy for the risk score and NT-proBNP in predicting all-cause mortality (HR 0.74, 95\% CI 0.65-0.81 vs. HR 0.74, 95\% CI 0.65-0.81, $p=0.99$ ) and CV mortality (HR 0.68, 95\% CI 0.59-0.76 vs. HR 0.67, 95\% CI 0.58-0.75, $p=0.95$ ). When the risk score was added to the NT-proBNP, the continuous net reclassification improvement was $56 \%$ for predicting all-cause mortality (95\% CI 18-95\%, $p=0.004)$ and $45 \%$ for predicting CV mortality (95\% CI 2-89\%, $p=0.040)$.

Conclusions: In HFrEF, a risk score that included age, TnT, and CysC showed efficacy similar to the NT-proBNP for predicting all-cause mortality and CV mortality in an older population. (Cardiol J 2015; 22, 1: 31-36)
\end{abstract}

Key words: heart failure, prognosis, cardiovascular mortality, biomarkers, risk score

Address for correspondence: Michael Lx Fu, MD, PhD, FESC, Institute of Medicine, Sahlgrenska University Hospital/Östra, 41345 Gothenburg, Sweden, tel: 0046 313428481, fax: 0046 31259254, e-mail: michael.fu@wlab.gu.se Received: 16.12.2013

Accepted: 13.01.2014 


\section{Introduction}

Approximately $1-2 \%$ of the population in the western world has heart failure (HF) [1]. The prevalence of $\mathrm{HF}$ exceeds $8 \%$ among individuals over 75 years old [1]. The annual mortality varies from 5\% [2] to 75\% in cases of end-stage HF [3]. This broad range indicates that the prognosis is extremely diverse and can be difficult to assess in individual patients. In older patients with $\mathrm{HF}$ that have a reduced ejection fraction ( $\mathrm{HFrEF}$ ), about two thirds of deaths have been attributed to cardiovascular (CV) causes [4]. Therefore, approximately one third of deaths are due to non-CV causes. These latter causes are probably related to the increasing rate of co-morbidity; thus, HF appears to be heterogeneous in older individuals.

We previously developed a prognostic model that aimed to account for individual variations and confounding factors due to comorbidity in older individuals. This model comprised 3 risk markers that were highly associated with all-cause mortality [5]. The model produced a risk score that reflected the severity of the 3 risk markers: age, serum troponin $\mathrm{T}(\mathrm{Tn} \mathrm{T})$, and serum cystatin $\mathrm{C}$ (CysC) [5]. Each risk marker defined as age $>75$ years, $\mathrm{Tn} \mathrm{T}>10 \mathrm{ng} / \mathrm{L}$ and $\mathrm{CysC}>1.3 \mathrm{mg} / \mathrm{L}$ adds 1 point respectively in the risk score with total score levels ranging from 0 (lowest risk) to 3 points (highest risk).

The present study aimed to evaluate this risk score in a validation cohort of older patients with HFrEF.

\section{Methods}

\section{Study cohort and diagnosis}

This prospective study included patients scheduled to undergo echocardiography at the Department of Clinical Physiology, Sahlgrenska University Hospital, Gothenburg, Sweden, in 2010. Eligible patients had a final diagnosis of HFrEF based on HF symptoms and a left ventricular ejection fraction $(\mathrm{LVEF})<50 \%$ [6]. Two patients were excluded from the study due to the lack of a Swedish identification number, which would make any follow-up impossible.

All patients underwent echocardiography, blood sampling, and a clinical examination within approximately $24 \mathrm{~h}$ after echocardiography. Echocardiograms were analyzed by an echocardiography specialist, independent of the study investigators, who was blinded to other variables in this study.

The study protocol was approved by the Ethics Committee at the University of Gothenburg.
Written informed consent was obtained from all the patients and the study protocol conformed to the ethical guidelines of the 1975 Declaration of Helsinki.

\section{Laboratory analyses}

The N-terminal prohormone of B-type natriuretic peptide (NT-proBNP), CysC, high-sensitivity-TnT and creatinine measurements were obtained from the routine laboratory services provided by the Clinical Chemistry Laboratory at Sahlgrenska University Hospital. The estimated glomerular filtration rate was calculated according to the Cockcroft-Gault formula. The performance of the laboratory assays was described previously $[7,8]$.

\section{Follow-up and outcomes}

All patients were followed up at 3 years. Data on the causes of death were obtained from the death registry of the National Board of Health and Welfare in Sweden. Outcome measures were all-cause mortality and CV mortality. The causes of death were classified according to the International Statistical Classification of Diseases and Related Health Problems, $10^{\text {th }}$ revision (ICD-10). The automatic coding of medical entities (ACME) system [9] was used to select the underlying cause of death. ACME used the ICD codes and their locations recorded on the death certificate for determining the cause of death. $\mathrm{CV}$ mortality was defined as an underlying cause of death that was classified as I05-I09 (chronic rheumatic heart diseases), I20-I51 (ischemic heart disease, pulmonary heart disease, diseases of pulmonary circulation, and other forms of heart disease), or I60-I69 (cerebrovascular diseases).

\section{Statistical analyses}

Kaplan-Meier analyses were used to evaluate possible associations between mortality and the risk scores. Hazard ratios (HR) with confidence intervals were calculated to evaluate risks. Univariate comparisons between dead and alive at 3 years were calculated with median tests. Dichotomous variables were analyzed with Monte-Carlo estimates. Receiver operating characteristic (ROC) curves were used to assess the prognostic properties of the risk score and the NT-proBNP classification. Areas under the ROC curves (AUC) were compared with the DeLong methodology [10]. Statistical analyses were performed with SPSS version 20 and Medcalc 12. All probabilities were 2 -tailed, and $\mathrm{p}$ values $<0.05$ were considered significant. NT-proBNP levels were classified based on 3 previously defined risk groups [5] (low risk 
Table 1. Baseline characteristics of patients that survived or died at the 3-year follow-up.

\begin{tabular}{|c|c|c|c|}
\hline Variable & Survived $(n=87)$ & $\operatorname{Died}(\mathbf{n}=37)$ & $\mathbf{P}$ \\
\hline \multicolumn{4}{|l|}{ Clinical data } \\
\hline Age & $72(63-78)$ & $78(71-84)$ & 0.20 \\
\hline Gender - male & $72 \%$ & $73 \%$ & 1.0 \\
\hline Systolic BP [mm Hg] (median + IQR) & $125(110-150)$ & $125(110-145)$ & 0.88 \\
\hline Diastolic BP [mm Hg] (median + IQR) & $75(65-87)$ & $70(70-80)$ & 0.70 \\
\hline \multicolumn{4}{|l|}{ Echocardiogram parameters } \\
\hline LVEF [\%] & $35(30-40)$ & $33(25-40)$ & 0.86 \\
\hline LVDD $[\mathrm{cm}]($ median + IQR) & $6(5-6)$ & $6(5-7)$ & 0.98 \\
\hline $\mathrm{PA}[\mathrm{mm} \mathrm{Hg}]$ (median + IQR) & $38(30-45)$ & $42(30-50)$ & 0.36 \\
\hline Stroke volume (median + IQR) & $53(44-65)$ & $52(41-59)$ & 1.0 \\
\hline \multicolumn{4}{|l|}{ Cardiovascular risk factors } \\
\hline Smoking & $30 \%$ & $35 \%$ & 0.67 \\
\hline Hypertension & $38 \%$ & $35 \%$ & 0.84 \\
\hline Diabetes mellitus & $20 \%$ & $35 \%$ & 0.071 \\
\hline \multicolumn{4}{|l|}{ Cardiovascular disease } \\
\hline Myocardial infarction & $45 \%$ & $43 \%$ & 1.0 \\
\hline Angina pectoris & $5.7 \%$ & $8.1 \%$ & 0.70 \\
\hline Atrial fibrillation & $38 \%$ & $35 \%$ & 0.84 \\
\hline \multicolumn{4}{|l|}{ Comorbidity } \\
\hline Pulmonary disease & $21 \%$ & $24 \%$ & 0.81 \\
\hline Stroke & $9.2 \%$ & $22 \%$ & 0.079 \\
\hline Renal failure (GFR < 60) & $35 \%$ & $73 \%$ & $<0.001$ \\
\hline \multicolumn{4}{|l|}{ Medications } \\
\hline ACE inhibitors & $52 \%$ & $32 \%$ & 0.052 \\
\hline Beta-blockers & $83 \%$ & $70 \%$ & 0.15 \\
\hline Diuretics & $51 \%$ & $84 \%$ & 0.001 \\
\hline \multicolumn{4}{|l|}{ Laboratory markers } \\
\hline S-creatinine $[\mu \mathrm{mol} / \mathrm{L}]($ median $+\mathrm{IQR})$ & $92(74-106)$ & $121(89-176)$ & 0.016 \\
\hline Estimated GFR $[\mathrm{mL} / \mathrm{min}]$ (median + IQR) & $75(56-98)$ & $43(29-62)$ & 0.001 \\
\hline Cystatin C [mg/L] (median + IQR) & $1.0(0.82-1.2)$ & $1.7(1.2-2.2)$ & $<0.001$ \\
\hline High-sensitivity-troponin T [ng/L] (median + IQR) & $24(8-63)$ & $49(24-105)$ & 0.016 \\
\hline NT-proBNP [ng/L] (median + IQR) & $1670(569-3410)$ & $5890(2200-20600)$ & 0.016 \\
\hline
\end{tabular}

ACE - angiotensin converting enzyme inhibitor; BP — blood pressure; GFR - glomerular filtration rate; IQR — interquartile range; LVDD - left ventricular end-diastolic diameter; LVEF — left ventricular ejection fraction; NT-proBNP - N-terminal pro B-type natriuretic peptide; PA - pulmonary artery

$<2000 \mathrm{ng} / \mathrm{L}$, medium risk $=2000-8000 \mathrm{ng} / \mathrm{L}$, and high risk $\geq 8000 \mathrm{ng} / \mathrm{L})$. Reclassification improvement was assessed with the continuous net reclassification improvement (NRI) method [11], because appropriate risk levels depend on the clinical situation and available resources. One patient (survivor at 3 years) was excluded from statistical analyses of laboratory data and the risk score due to the lack of available laboratory results.

\section{Results}

\section{Baseline characteristics}

The study participants comprised a representative population of individuals with HFrEF. They had a median age of 73 years (interquartile range
64-80 years). Baseline characteristics for patients that had died or survived at the 3-year follow up are presented in Table 1.

\section{All-cause and cardiovascular mortality}

In this cohort, all-cause mortality was $30 \%$ and $\mathrm{CV}$ mortality was $21 \%$. The risk score was able to separate individuals with low risk (0-1 score points) from those with moderate-high risk (2-3 score points) for all-cause mortality (HR 4.2, 95\% confidence interval [CI] 2.2-8.1, p < 0.001, 0-1 vs. 2-3 score points; Fig. 1) and for CV mortality (HR 3.6, 95\% CI 1.7-8.0, $\mathrm{p}=0.0015,0-1$ vs. $2-3$ score points; Fig. 2). The probabilities of survival for patients in the 3 different classes of NT-proBNP are presented in Figures 3 and 4 for comparison. 


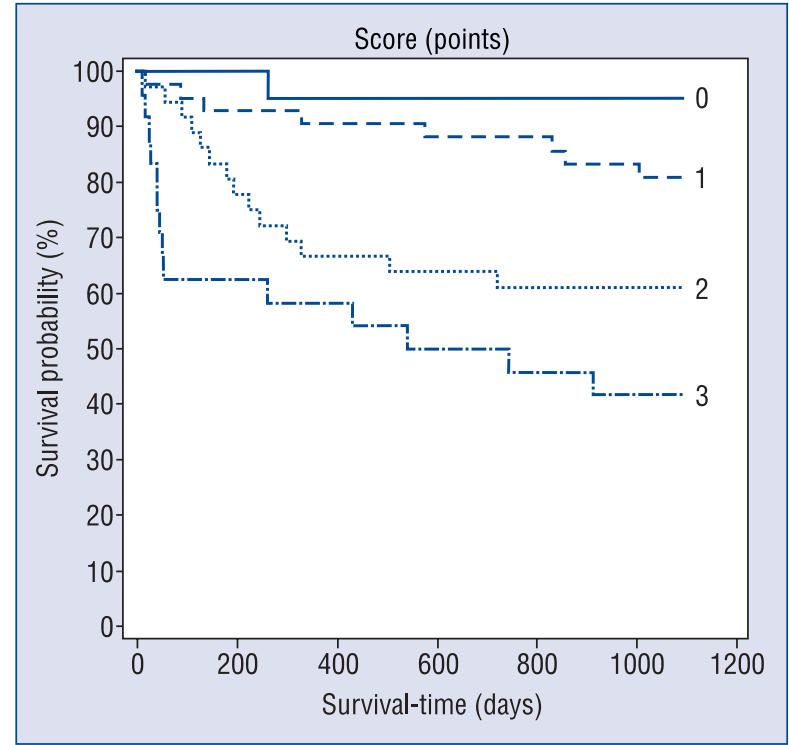

Figure 1. Association between the risk score and all-cause mortality. The risk score ranged from 0 to 3 points. The lines indicate the survival probability for individuals with the indicated scores, over the 3-year study period; $p<0.001$ for comparison between individuals with $0-1$ vs. $2-3$ points.

There was no difference in the AUCs between the predictions assessed with the risk score and those assessed with the NT-proBNP for either all-cause mortality (HR $0.74,95 \%$ CI $0.65-0.81$ vs. HR $0.74,95 \%$ CI $0.65-0.81, \mathrm{p}=0.99$ ) or $\mathrm{CV}$ mortality (HR $0.68,95 \%$ CI $0.59-0.76$ vs. HR 0.67 , $95 \%$ CI $0.58-0.75, \mathrm{p}=0.95)$. However, when the risk score was added to the NT-proBNP classification, the NRI for predicting all-cause mortality was $56 \%$ (95\% CI 18-95\%, $\mathrm{p}=0.004$ ) and the NRI for predicting CV mortality was $45 \%$ (95\% CI $2-89 \%$, $\mathrm{p}=0.040)$.

\section{Subgroup analyses of patients with}

NT-proBNP $<2500 \mathrm{ng} / \mathrm{L}$ and $\geq 2500 \mathrm{ng} / \mathrm{L}$

The risk score was able to differentiate risk levels in a subgroup of patients with NT-proBNP $<2500 \mathrm{ng} / \mathrm{L}$. Two groups were distinguished for all-cause mortality ( $\mathrm{p}<0.046$; scores $0-1$ vs. $2-3$ points) and for CV mortality ( $\mathrm{p}=0.040$; scores $0-1$ vs. $2-3$ points). In a subgroup of patients with NT-proBNP $\geq 2500 \mathrm{ng} / \mathrm{L}$, the risk score also differentiated two risk groups for all-cause mortality ( $p<0.021$; scores $0-1$ vs. $2-3$ points), but not for CV mortality ( $\mathrm{p}=0.13$; scores $0-1$ vs. $2-3$ points).

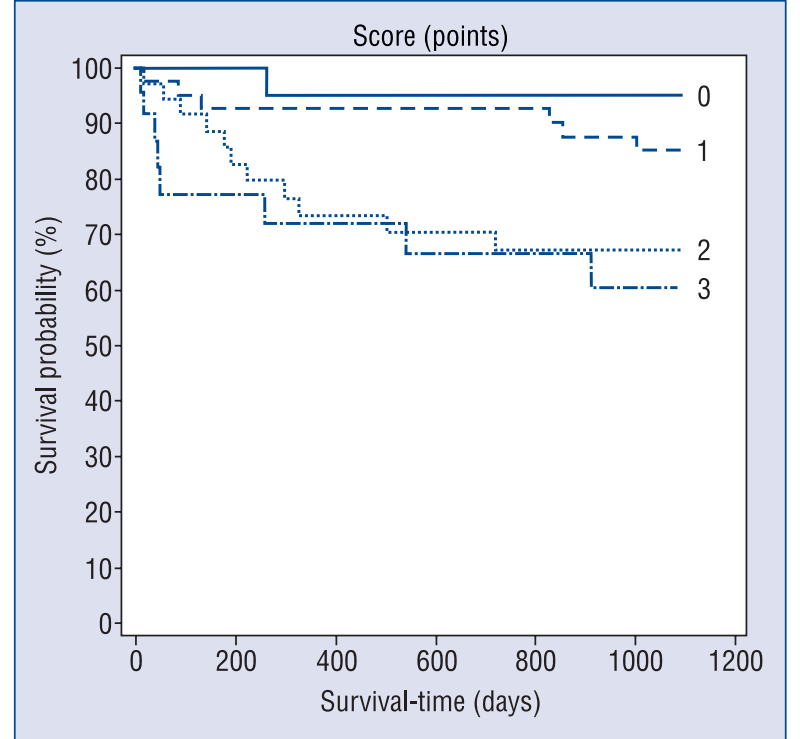

Figure 2. Association between the risk score and cardiovascular mortality. The risk score ranged from 0 to 3 points. The lines indicate the survival probability for individuals with the indicated scores, over the 3-year study period; $p=0.0015$ for comparison between individuals with $0-1$ vs. $2-3$ points.

\section{Discussion}

This study examined a real-life cohort of older patients with HF to assess our previously developed multi-marker prognostic risk score, which included age, TnT, and CysC [5]. Our present results showed that the efficacy of our risk score was similar to that of the NT-proBNP classification for predicting all-cause mortality and CV mortality. Moreover, a combination of both the risk score and the NT-proBNP classification provided higher prognostic value than the NT-proBNP classification alone, for both all-cause and CV mortality.

In our cohort, approximately $70 \%$ of patients died from CV causes. This was consistent with results from previous studies. Henkel et al. [4] found that CV mortality constituted $64 \%$ of all deaths in a cohort aged $75.0 \pm 12.7$ years with HFrEF. Another study by Mehta et al. [11] showed that CV mortality constituted $86 \%$ of all deaths in a cohort aged $75.2 \pm 12.4$ years.

The present validation cohort was different from our previous HF cohort in 3 important aspects: (1) This study was conducted prospectively in patients scheduled to undergo echocardio- 


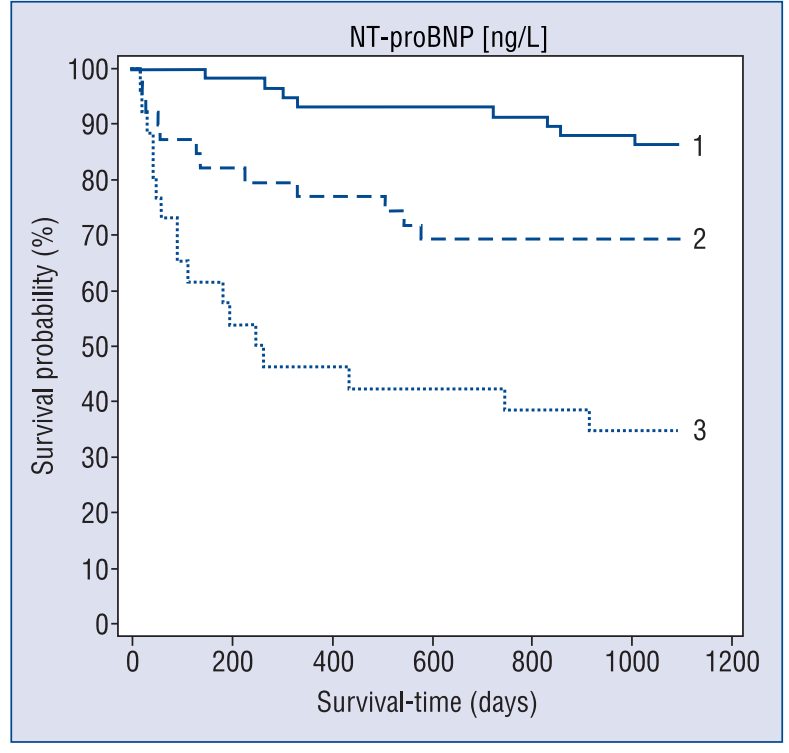

Figure 3. Association between different $\mathrm{N}$-terminal pro B-type natriuretic peptide (NT-proBNP) risk levels (low risk $<2000 \mathrm{ng} / \mathrm{L}$, medium risk $=2000-8000 \mathrm{ng} / \mathrm{L}$, and high risk $\geq 8000 \mathrm{ng} / \mathrm{L}$ ) and all-cause mortality $(p<0.001)$.

graphy due to clinically suspected HF. Therefore, there was a short time delay between echocardiography and biomarker analyses; (2) Patients with LVEF $\geq 50 \%$ were excluded in this validation cohort; (3) The present validation cohort consisted of patients referred to echocardiography both from the hospital and from primary care; in contrast, the score was previously developed with patients in hospital care. Despite these differences in cohorts, the risk score remained effective for predicting adverse outcomes.

Compared to the NT-proBNP classification, the score had several main advantages. (1) It provided complementary prognostic information to the NT-proBNP for predicting both all-cause and CV-mortality, as indicated by the NRI; (2) The risk score could stratify risk among patients with relatively low levels of NT-proBNP $(<2500 \mathrm{ng} / \mathrm{L})$. Among this group of patients, the all-cause mortality was $35 \%$ different between patients with scores of $0-1$ points vs. those with scores of $2-3$ points (data not shown). Precise prognostic information is important for tailoring treatment to individual patients and/or for facilitating the allocation of limited health care resources; (3) It could be argued that the risk score may be less influenced than NT-proBNP levels by analytical and intra-individual variations $[12,13]$, which might compromise the

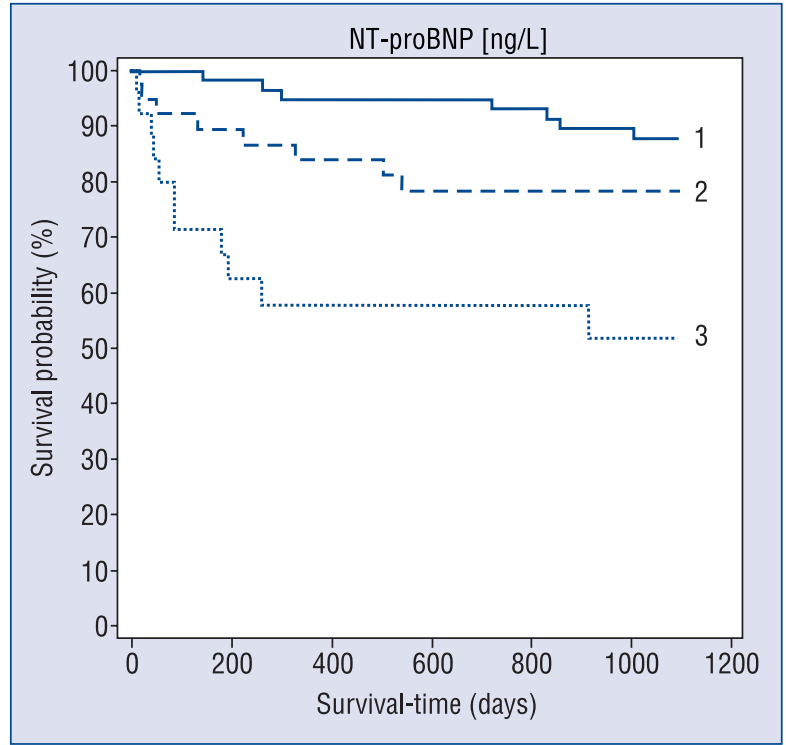

Figure 4. Association between different $\mathrm{N}$-terminal pro B-type natriuretic peptide (NT-proBNP) risk levels (low risk $<2000 \mathrm{ng} / \mathrm{L}$, medium risk $=2000-8000 \mathrm{ng} / \mathrm{L}$, and high risk $\geq 8000 \mathrm{ng} / \mathrm{L}$ ) and cardiovascular mortality ( $p<0.001)$.

accuracy of prognostic assessments; (4) The risk score takes into account renal function and high-sensitivity TnT levels, both known markers of risk in HF. In fact, CysC has been shown to be a stronger predictor of mortality than creatinine in older patients with HF [14]. Moreover, de Antonio et al. [15] found that high-sensitivity TnT provided prognostic information in patients with $\mathrm{HF}$ with a median age of 70.3 years, even after adjustment for NT-proBNP and other risk factors.

Our proposed risk score is simpler to apply than, for instance, the Seattle Heart Failure Model [16] and the MAGGIC risk score [17]. This facility might encourage broader implementation, because busy clinicians must typically prioritize multiple tasks. Therefore, we anticipate that our suggested risk score may be a valuable tool in clinical practice in the future. However, one potential weakness in this study was a rather small sample size.

\section{Conclusions}

In HFrEF, a risk score that included age, TnT, and CysC had an accuracy similar to that of the NT-proBNP classification for predicting both all-cause mortality and CV mortality. A combination of the NT-proBNP and the risk score provided high prognostic value for predicting both all-cause and CV mortality. 


\section{Acknowledgements}

This work was supported by the Heart and Lung Foundation. We also thank the staff at the Department of Clinical Chemistry at Sahlgrenska University Hospital for their expertise and their analyses of the laboratory parameters.

\section{Conflict of interest: None declared}

\section{References}

1. Mosterd A, Hoes AW. Clinical epidemiology of heart failure. Heart, 2007; 93: 1137-1146.

2. Aaronson KD, Schwartz JS, Chen TM, Wong KL, Goin JE, Mancini DM. Development and prospective validation of a clinical index to predict survival in ambulatory patients referred for cardiac transplant evaluation. Circulation, 1997; 95: 2660-2667.

3. Rose EA, Gelijns AC, Moskowitz AJ et al. Long-term use of a left ventricular assist device for end-stage heart failure. $\mathrm{N} \mathrm{Engl}$ J Med, 2001; 345: 1435-1443.

4. Henkel DM, Redfield MM, Weston SA et al. Death in heart failure: a community perspective. Circ Heart Fail, 2008; 1: 91-97.

5. Bjurman C, Jensen J, Petzold M, Hammarsten O, Fu ML. Assessment of a multimarker strategy for prediction of mortality in older heart failure patients: a cohort study. BMJ Open 2013; 3: pii: e002254.

6. Dickstein K, Cohen-Solal A, Filippatos G et al. ESC Guidelines for the diagnosis and treatment of acute and chronic heart failure 2008. Eur Heart J, 2008; 29: 2388-2442.

7. Hammarsten O, Jacobsson CE, Widegren M, Danylchenko T, Jaffe AS. Long-time quality assessment of the Elecsys Troponin T hs assay. Clin Biochem, 2013; 46: 1055-1057.

8. National Center for Health Statistics. The Mortality Medical Data System, MICAR, ACME and TRANSAX. NCHS, Hyattsville, MD 1990.
9. DeLong ER, DeLong DM, Clarke-Pearson DL. Comparing the areas under two or more correlated receiver operating characteristic curves: A nonparametric approach. Biometrics, 1988; 44: 837-845.

10. Pencina MJ, D’Agostino RB, Steyerberg EW. Extensions of net reclassification improvement calculations to measure usefulness of new biomarkers. Stat Med, 2011; 30: 11-21.

11. Mehta PA, Dubrey SW, McIntyre HF et al. Mode of death in patients with newly diagnosed heart failure in the general population. Eur J Heart Fail, 2008; 10: 1108-1116.

12. Bruins S, Fokkema MR, Römer JW et al. High intraindividual variation of B-type natriuretic peptide (BNP) and amino-terminal proBNP in patients with stable chronic heart failure. Clin Chem, 2004; 50: 2052-2058.

13. Clerico A, Carlo Zucchelli G, Pilo A, Passino C, Emdin M. Clinical relevance of biological variation: the lesson of brain natriuretic peptide (BNP) and NT-proBNP assay. Clin Chem Lab Med, 2006; 44: $366-378$.

14. Shlipak MG, Katz R, Fried LF et al. Cystatin-C and mortality in elderly persons with heart failure. J Am Coll Cardiol, 2005; 45: 268-271.

15. de Antonio M, Lupon J, Galan A, Vila J, Urrutia A, Bayes-Genis A. Combined use of high-sensitivity cardiac troponin $\mathrm{T}$ and N-terminal pro-B type natriuretic peptide improves measurements of performance over established mortality risk factors in chronic heart failure. Am Heart J, 2012; 163: 821-828.

16. Levy WC, Mozaffarian D, Linker DT et al. The Seattle Heart Failure Model: prediction of survival in heart failure. Circulation, 2006; 113: 1424-1433.

17. Pocock SJ, Ariti CA, McMurray JJ et al. Predicting survival in heart failure: A risk score based on 39372 patients from 30 studies. Eur Heart J, 2013; 34: 1404-1413. 